\title{
Acute pulmonary embolism in a young patient with deep vein thrombosis
}

\begin{abstract}
Pulmonary embolism (PE) is a potential cardiovascular emergency caused by part of a thrombus, usually dislodged from a deep vein thrombosis (subsequently called an embolus), passing into the pulmonary circulation and preventing blood flow to the lungs. The condition is frequently termed pulmonary thrombo-embolism. PE is one of the most common reason for preventable hospital death. A young adult presenting with acute onset of dyspnea, pulmonary embolism should be kept a possibility, as most of the time PE is not considered in differential diagnosis while evaluating such patients in emergency. Most of young patients reported in literature are young females, either pregnant or on oral contraceptives. Its need high clinical suspicion for diagnosis of acute pulmonary embolism. There is one case reports of young male, who presented as acute onset of breathlessness and subsequently diagnosed and treated as a case of pulmonary thromboembolism.
\end{abstract}

Keywords: pulmonary embolism, deep vein thrombosis
Volume 4 Issue 6 - 2017

\author{
Sharzia Asma-Ul Hosna,' Mahesh \\ Chaudhary, ${ }^{2}$ Mohammad Amdadul Haque ${ }^{3}$ \\ 'LABAID Specialized Hospital, Bangladesh \\ ${ }^{2}$ Department of Radiology \& Imaging, Bangabandhu Sheikh Mujib \\ Medical University, Bangladesh \\ ${ }^{3}$ Department of Cardiology, Bangladesh
}

\begin{abstract}
Correspondence: Sharzia Asma-UI Hosna, LABAID Specialized Hospital, Dhaka, Bangladesh, Tel 00880181479250I, Email sharzia.amey@gmail.com
\end{abstract}

Received: November 30, 2017 | Published: December 20, 2017
Abbreviations: ICU, intensive care unit; LMWH, low molecular weight heparin; PTE, pulmonary thrombo embolism; RV, right ventricle; ELISA, enzyme-linked immunosorbent assay; CTPA, ct pulmonary angiography; UFH, unfractioned heparin

\section{Introduction}

Pulmonary embolism remains a disease which needs early diagnosis \& management to prevent mortality and morbidity. Awareness regarding PE is lower than that for other thrombotic disorders, such as heart attack and stroke. ${ }^{1}$ Incidence of pulmonary embolism is around $0.5-1$ case per $1000 .^{2}$ Study shows that, about $70 \%$ of proven post mortem cases of pulmonary embolism are not even suspected during the course of treatment. ${ }^{3}$ Young patients are more likely to be mismanaged, as suspicion in healthier young patient is very low.

\section{Case report}

A non-diabetic, non-hypertensive 34year-old young male patient, presented to emergency department with history of cough for 5days and breathlessness on exertion for 3days. Physical examination revealed no significant finding except mild edema on left leg. Patient is a chain smoker. Initial lab work showed White blood cell count of $12000 / \mathrm{mcl}$, platelets $410,000 / \mathrm{mcl}$, and hemoglobin $12.8 \mathrm{gm} / \mathrm{dl}$. Metabolic profile, liver function tests and renal function tests were normal. Chest x-ray was normal. Echocardiography revealed features of pulmonary hypertension. After resuscitation patient was shifted to intensive care unit (ICU) for further management. Doppler study was advised for left lower leg edema which revealed occlusive DVT of the left common femoral, superficial, deep femoral \& popliteal vein. After that clinical suspicion for pulmonary embolism was suspected $\&$ he was advised for CT pulmonary angiography. A CT pulmonary angiography demonstrated emboli in right main pulmonary artery, $\&$ in main, segmental branch of left pulmonary artery with wedge shape area of pulmonary infarct on left lower lobe. ECG finding was consistent with a new onset atrial fibrillation, which is highly suggestive for new onset PE. After final diagnosis low molecular weight heparin (LMWH) was started associated with other supportive care. Patient improved gradually over a period of 1 week with normal pulse and respiratory rate. ECG improved with normal sinus rhythm. Patient was put on oral coagulant and discharged with advice for follow-up (Figure 1-4).

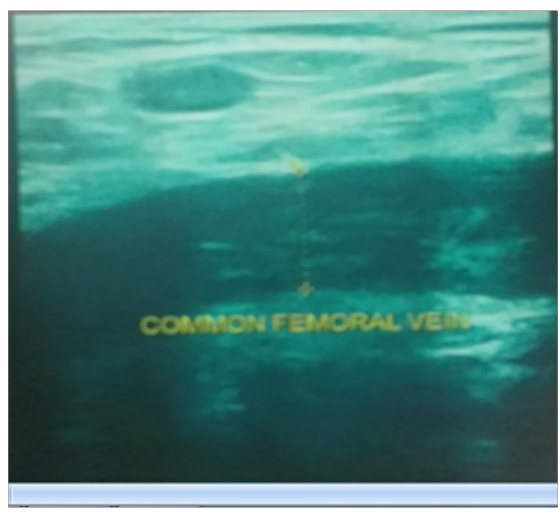

Figure I USG shows mixed echogenic structure occluding common left femoral vein, suggestive of thrombus.

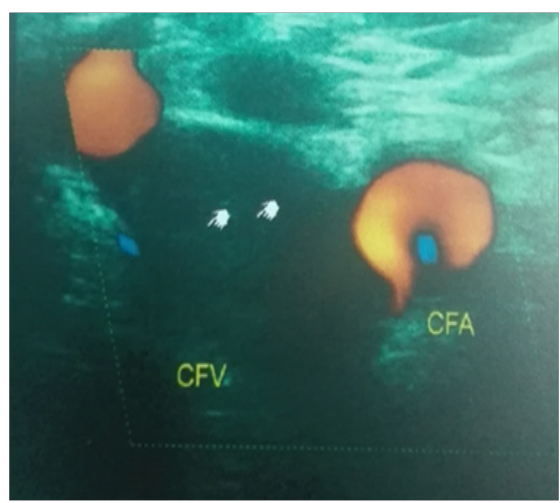

Figure 2 Color doppler USG shows absent flow within common left femoral vein due to occluding thrombus. 


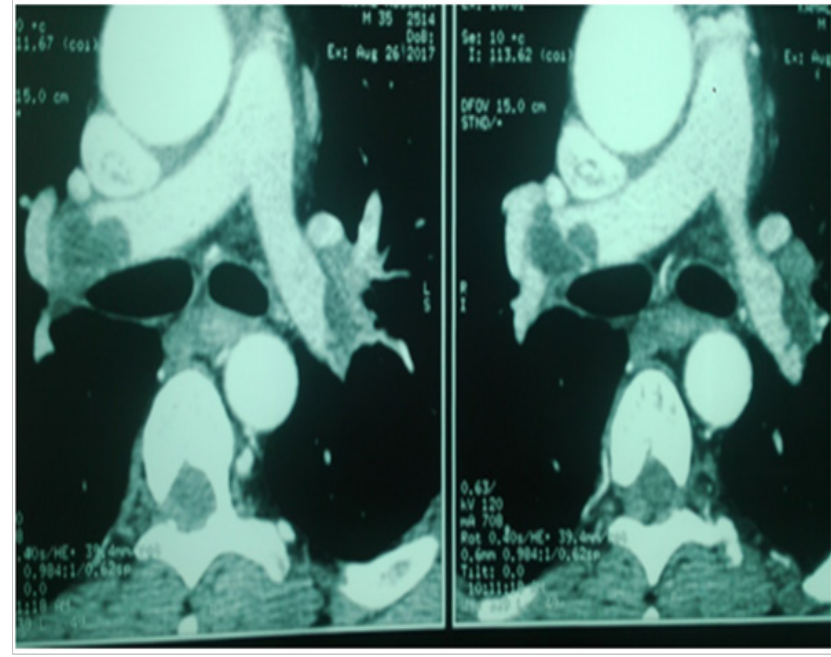

Figure 3 CT pulmonary angiography showing filling defect within both main pulmanary artery, suggest emboli.

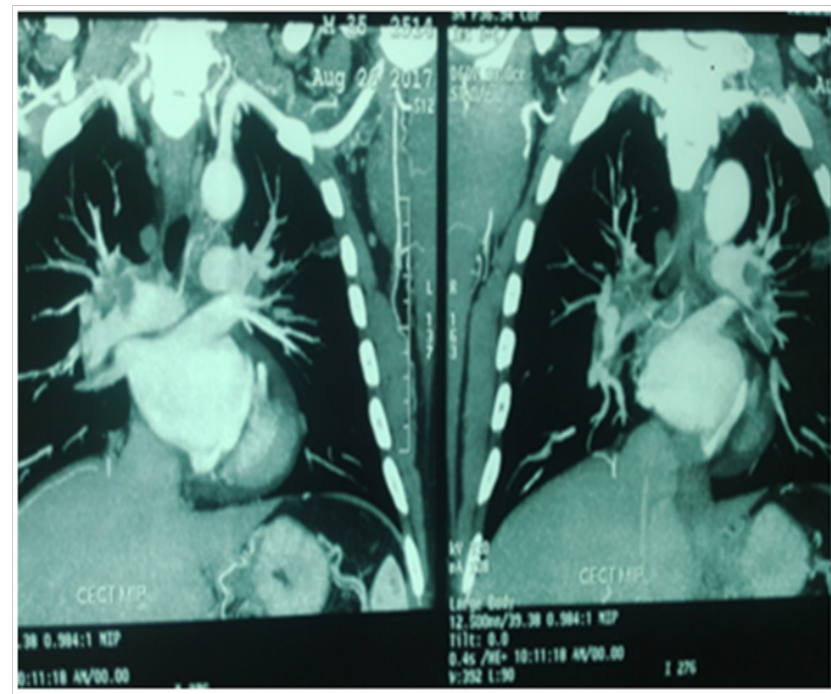

Figure 4 Coronal section of CT pulmonary angiography, showing emboli within segmental branch of right \& left pulmonary artery.

\section{Discussion}

PE is a potentially life threatening condition, and in severe case the occurrence of circulatory collapse and cardiac arrest may result in sudden death. Around $80 \%$ of patients with PE have signs of DVT, and approximately $50 \%$ of patients with proven proximal DVT have an associated PE. ${ }^{4}$ Thrombosis is caused by hypercoagulability, trauma and stasis. Recent surgery, trauma, immobilization, pregnancy and oral contraceptives are commonest acquired. ${ }^{5}$ Other factors like, malignancies for example hematological, lung, and pancreatic and brain cancer has risk for pulmonary thrombo embolism (PTE). Smoking, obesity, atherosclerosis, hypertension and infection in hospitalized patients are also common causes for PTE. Dislodged embolus from the lower part of the body pass through the inferior vena cava, to right atrium, to right ventricle, to the pulmonary artery. This might eventually obstruct blood flow to the lung.

\section{Diagnosis}

Early fatality occurs in up to $15 \%$ of patients; therefore, rapid diagnosis is crucial. Similar to DVT, PE is often asymptomatic.
Common signs and symptoms of PE are dyspnea, pleuritic chest pain, cough, sub sternal chest pain, fever, hemoptysis, syncope, unilateral leg pain and signs of DVT (unilateral extremity swelling). CXR is useful to rule out other causes of dyspnea. But most of the time chest $\mathrm{x}$-ray revels normal. Other findings on chest x-ray are Hampton's hump sign (subpleural wedge shape opacity), Westermarck's sign (areas of hyperlucency), raised hemi diaphragm, pleural effusion. ${ }^{6}$ ECG may show T wave inversion S1Q3T3 pattern in around 50\% of cases, which denotes right ventricle (RV) damage. Sinus tachycardia is present in around $40 \%$ of cases. ${ }^{.} \mathrm{D}$-dimer (ELISA) is commonly used as a screening test in patients with a low and moderate probability clinical assessment. Normal D-dimer has almost $100 \%$ negative predictive value (virtually excludes PE), no further testing is required. CT pulmonary angiography is the preferred method of diagnostic imaging in patients with $\mathrm{PE}$, because it provides a high resolution image and is as accurate and less invasive. ${ }^{8} \mathrm{CT}$ pulmonary angiography (CTPA) will show filling defects within the pulmonary vasculature with acute pulmonary emboli. When observed in the axial plane this has been described as the polo mint sign. CTPA angiography for diagnosis of pulmonary embolism has sensitivity of $83 \%$ and specificity of $96 \% .{ }^{9}$ 2-D ECHO revealed features of PAH. $\mathrm{RV}$ dilatation, presence of thrombi in $\mathrm{RV}$, isother important finding in 2-D ECHO. Doppler study of lower limb shows DVT in 30-50\%of patient.

LMWH anticoagulants such as Enoxaparin, Dalteparin, Tinzaparin and Fondaparinux are preferred over unfractioned heparin (UFH), as chances of bleeding and Heparin-Induced Thrombocytopenia (HIT) are very low, and monitoring is not required except in special circumstances such as pregnancy in which anti Factor Xa activity is monitored. ${ }^{10}$

\section{Conclusion}

Pulmonary embolism in young adults is not very uncommon. A young adult presenting with acute onset of dyspnea, pulmonary embolism should be kept as a possible cause. Diagnosis of pulmonary embolism requires high clinical suspicion followed by multi-modality investigation. Unlike infectious diseases, cardiac disorder or blood disorder, no specific symptoms, signs or investigations reflect a disease process immediately. Anticoagulation is provided in patients without risk of active bleeding. If the emboli are large or there is a large clot burden, thrombolysis is an option. Hence, young male presenting with acute onset of dyspnoea should be evaluated for common cause of dyspnea but pulmonary embolism should be kept in mind, so as not to miss this fatal disease.

\section{Acknowledgements}

None.

\section{Conflict of interest}

Author declares that there is no conflict of interest.

\section{References}

1. Wendelboe AM, McCumber M, Hylek EM, et al. Global public awareness of venous thromboembolism. Journal of Thrombosis and Haemostasis. 2015;13:1365-1371.

2. Heit JA. The epidemiology of venous thromboembolism in the community. Arteroscler Thromb Vasc Biol. 2008;28(3):370-372.

3. Goldhaber SZ, Hennekens CH, Evans DA, et al. Factors associated with correct antemortem diagnosis of major pulmonary embolism. Am J Med. 1982;73(6):822-826. 
4. Anderson FA, Spencer FA. Risk factors for venous thromboembolism Circulation. 2003;107:19-16.

5. Stein PD, Woodard PK, Weg JG, et al. Diagnostic pathways in acute pulmonary embolism: recommendations of the PIOPED II Investigators. Radiology. 2007;242(1):15-21.

6. Elliot CG, Goldhaber SZ, Visani L, et al. Chest radiography in acute pulmonary embolism. Results from the International Cooperative Pulmonary Embolism Registry. Chest. 2000;118(1):33-38.

7. Ferrari E, Imbert A, Chevalier T, et al. The ECG in pulmonary embolism. Predictive value of negative T waves in precordial leads -80 case reports. Chest. 1997;111(3):537-543.
8. Konstantinides SV, Torbicki A, Agnelli G, et al. ESC Guidelines on the diagnosis and management of acute pulmonary embolism. Eur Heart J. 2014;35:3033-3069.

9. Stein PD, Fowler SD, Goodman LR, et al. Multi detector computed tomography for acute pulmonary embolism. $N$ Engl $\mathrm{J} \mathrm{Med}$. 2006;354(22):2317-2327.

10. Cossete B, Pelletier ME, Carrier N, et al. Evaluation of bleeding risk in patients exposed to therapeutic unfractioned or low molecular weight heparin: A cohort study in context of a quality improvement initiative. Ann Pharmacother. 2010;44(6):994-1002. 